\title{
UNSUPERVISED TEXTURE SEGMENTATION USING MONOGENIC CURVELETS AND THE POTTS MODEL
}

\author{
Martin Storath $^{1}$, Andreas Weinmann ${ }^{2}$, Michael Unser ${ }^{1}$ \\ ${ }^{1}$ Biomedical Imaging Group, École polytechnique fédérale de Lausanne (EPFL), Switzerland \\ ${ }^{2}$ Department of Mathematics, TU München and Helmholtz Zentrum München, Germany
}

\begin{abstract}
We present a method for the unsupervised segmentation of textured images using Potts functionals, which are a piecewise-constant variant of the Mumford and Shah functionals. We propose a minimization strategy based on the alternating direction method of multipliers and dynamic programming. The strategy allows us to process large feature spaces because the computational cost grows only linearly in the feature dimension. In particular, our algorithm has more favorable computational costs for high-dimensional data than graph cuts. Our feature vectors are based on monogenic curvelets. They incorporate multiple resolutions and directional information. The advantage over classical curvelets is that they yield smoother amplitudes due to the envelope effect of the monogenic signal.
\end{abstract}

Index Terms - Texture segmentation, Potts functional, piecewise constant Mumford and Shah functional, monogenic curvelets.

\section{INTRODUCTION}

The problem of segmenting textured images is a basic, yet challenging, image-processing task which appears in various contexts. Examples of recent papers on the topic are $[1,2]$ in biomedical imaging as well as $[3,4]$ in computer vision. The common strategy is to first create a feature vector for each pixel of the image. This yields a multicomponent image of local texture features that is then used to drive the segmentation process.

There is a multitude of approaches to the determination of texture features of which we shall only mention the primary ones. The early ones are based on local gray-level cooccurences and autocorrelations, as documented in the survey [5]. In many articles, filtering approaches such as Gabor filters [6] and wavelet frames [7] are used. (We refer to [8] for a comparative study and for many additional references.)

The research leading to these results has received funding from the European Research Council under the European Union's Seventh Framework Programme (FP7/2007-2013) / ERC grant agreement no. 267439 and the German Federal Ministry for Education and Research under SysTec Grant 0315508.
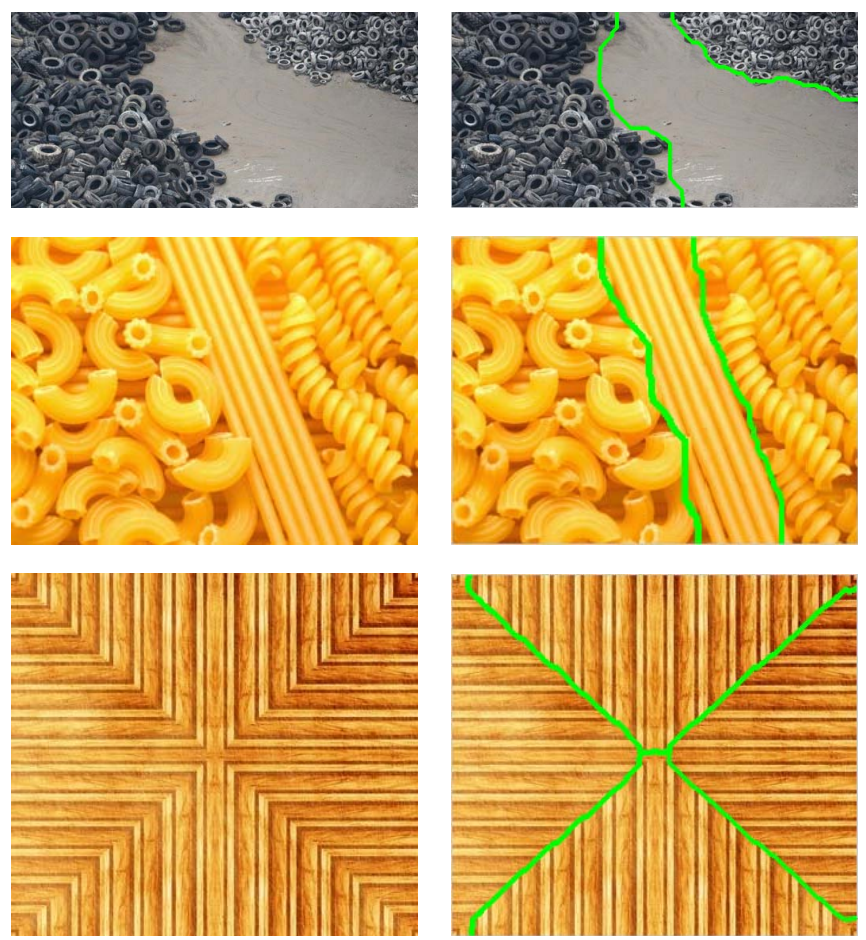

Fig. 1: Segmentation of textured images using our method.

Further approaches are based on local binary patterns [4] and structure tensors with nonlinear diffusion [9]. The second step is the segmentation itself. Classical techniques are K-means clustering $[6,7]$ and vector quantization [8]. More recent works employ the mean-shift algorithm [10], level-set methods [9], or minimization of convex energy functionals [11].

We here rely on the Potts model $[12,13]$ which is frequently used in the context of unsupervised image segmentation. Its continuous domain formulation is given by

$$
u^{*}=\arg \min _{u} \gamma\|\nabla u\|_{0}+\|u-F\|_{2}^{2} .
$$

The data $F$ is a multicomponent image taking values in $\mathbb{R}^{s}$ and the data fidelity is measured by an $L^{2}$ norm. The labeling function $u$ is piecewise constant. Its jump or discontinuity set encodes the boundaries of the corresponding segmentation. The symbol $\|\nabla u\|_{0}$ denotes the total length of the 
segment boundaries induced by $u$. The empirical model parameter $\gamma>0$ controls the balance between data fidelity and the regularizing term. A state-of-the-art minimization strategy is based on graph cuts [14]. This type of algorithm has also been deployed for texture segmentation [15]. The major drawback of graph cuts is that they become computationally demanding for large feature vectors. In fact, the complexity grows exponentially in the dimension $s$ of the feature vector. This makes graph cuts less appealing for texture segmentation since the feature vectors are typically high-dimensional in order to get good discriminative power. To circumvent this problem, the authors of [15] manually preselect between two and four feature components before applying the graph cuts algorithm.

In this paper, we propose a minimization strategy for the Potts functional which is computationally attractive even for high-dimensional feature vectors. Its computational costs grow only linearly in the dimension of the feature space. This allows us to process feature vectors of dimensions beyond 150 (as we did in Figure 1). Our strategy combines an ADMM splitting of a discretization of (1) and dynamic programming. We also propose to use feature vectors made of amplitudes of monogenic curvelet coefficients [16]. Curvelets are a family of multiscale atoms which increase their anisotropy at fine scales [17]. They have been used successfully as feature vectors $[18,19]$. The central advantage of monogenic curvelets over classical curvelets is that the amplitudes of the coefficients are less affected by ringing effects. This is due to the envelope effect of the monogenic signal $[20,16]$.

Our article is organized as follows: In Section 2, we study Potts functionals. We describe a fast algorithm that deals with high-dimensional feature vectors. In Section 3, we explain how to derive feature vectors using monogenic curvelets. Finally, we show the performance of the proposed method for texture mosaics as well as for natural images in Section 4.

\section{THE POTTS MODEL FOR UNSUPERVISED SEGMENTATION}

In this section we present a fast computational approach to the Potts problem (1) for vector-valued images. The approach is particularly suitable for high-dimensional feature vectors. We first discretize the jump term $\|\nabla u\|_{0}$ in (1) by

$$
\|\nabla u\|_{0}=\sum_{i, j} \sum_{(a, b) \in \mathcal{N}} \omega_{a, b}\left[u_{i, j,:} \neq u_{i+a, j+b,:}\right] .
$$

Here $u_{i, j,:}$ is a vector in $\mathbb{R}^{s}$ sitting at the pixel $(i, j)$ and $[v \neq w]$ denotes the Iverson bracket which takes value 0 if $v_{k}=w_{k}$ for all $k=1, \ldots, s$, and value 1 otherwise. The neighborhood relation $\mathcal{N}$ and the nonnegative weights $\omega_{a, b}$ define a discrete boundary length of the corresponding discrete partitions. For $\mathcal{N}=\{(0,1),(1,0)\}$ and $\omega_{0,1}=\omega_{1,0}=$ 1 , we get the simple (anisotropic) discretization which cor- responds to $\nabla u_{i j}=\left(u_{i j}-u_{i, j-1}, u_{i j}-u_{i-1, j}\right)$. This discretization measures the boundary length of the partitions in the (anisotropic) Manhattan metric. For our experiments, we use the 8-neighborhood $\mathcal{N}=\{(1,0),(0,1),(-1,1),(1,1)\}$ with diagonal weights $\omega_{1,1}=\omega_{-1,1}=\left(1-\frac{\sqrt{2}}{2}\right)$, and vertical and horizontal weights $\omega_{1,0}=\omega_{0,1}=(\sqrt{2}-1)$. The reason for using discretizations of the form (2) is that, in contrast to the simple discretization, they produce visually more pleasing and more isotropic reconstructions. Using this discrete version of $\|\nabla u\|_{0}$, we rewrite the discrete Potts functional as

$$
\begin{gathered}
u^{*}=\underset{u \in \mathbb{R}^{m \times n \times s}}{\operatorname{argmin}}\left\{\gamma \sum_{i, j} \sum_{(a, b) \in \mathcal{N}} \omega_{a, b} \cdot\left[u_{i, j,:} \neq u_{i+a, j+b,:}\right]\right. \\
\left.+\sum_{i, j, k}\left(u_{i, j, k}-F_{i, j, k}\right)^{2}\right\}
\end{gathered}
$$

Here, the data is given by the $m \times n$ feature image $F$ which takes its values in $\mathbb{R}^{s}$.

Our strategy for solving problem (3) is as follows. We consider the consensus form (see [21]) of (3), construct the augmented Lagrangian, and iteratively minimize for one of the respective variables while keeping the others fixed. Then, after some manipulations, we get the algorithm

$$
\left\{\begin{array}{l}
u^{k+1}=\arg \min _{u} \frac{4 \gamma \omega_{c}}{1+6 \mu}\left\|\nabla_{1} u\right\|_{0}+\left\|u-u^{\prime}\right\|_{2}^{2}, \\
w^{k+1}=\arg \min _{w} \frac{4 \gamma \omega_{d}}{1+6 \mu}\left\|\nabla_{12} w\right\|_{0}+\left\|w-w^{\prime}\right\|_{2}^{2} \\
v^{k+1}=\arg \min _{v} \frac{4 \gamma \omega_{c}}{1+6 \mu}\left\|\nabla_{2} v\right\|_{0}+\left\|v-v^{\prime}\right\|_{2}^{2} \\
z^{k+1}=\arg \min _{z} \frac{4 \gamma \omega_{d}}{1+6 \mu}\left\|\nabla_{21} z\right\|_{0}+\left\|z-z^{\prime}\right\|_{2}^{2}, \\
\lambda_{i}^{k+1}=\lambda_{i}^{k}+\mu a_{i}
\end{array}\right.
$$

where $\omega_{d}=\left(1-\frac{\sqrt{2}}{2}\right)$ and $\omega_{c}=(\sqrt{2}-1)$. The symbols $\nabla_{1}, \nabla_{2}, \nabla_{12}, \nabla_{21}$ denote the differences with respect to the vectors $(1,0),(0,1),(-1,1),(1,1)$. Here, the data are

$$
\begin{aligned}
u^{\prime} & =r\left[f+2 \mu\left(v^{k}+w^{k}+z^{k}\right)+2\left(-\lambda_{1}^{k}-\lambda_{2}^{k}-\lambda_{3}^{k}\right)\right], \\
w^{\prime} & =r\left[f+2 \mu\left(u^{k+1}+v^{k}+z^{k}\right)+2\left(\lambda_{2}^{k}+\lambda_{4}^{k}-\lambda_{6}^{k}\right)\right], \\
v^{\prime} & =r\left[f+2 \mu\left(u^{k+1}+w^{k+1}+z^{k}\right)+2\left(\lambda_{1}^{k}-\lambda_{4}^{k}-\lambda_{5}^{k}\right)\right], \\
z^{\prime} & =r\left[f+2 \mu\left(u^{k+1}+v^{k+1}+w^{k+1}\right)+2\left(\lambda_{3}^{k}+\lambda_{5}^{k}+\lambda_{6}^{k}\right)\right],
\end{aligned}
$$

where $r=\frac{1}{1+6 \mu}$. The updates $a_{i}$ are given by

$$
\begin{array}{ll}
a_{1}=u^{k+1}-v^{k+1}, & a_{2}=u^{k+1}-w^{k+1} \\
a_{3}=u^{k+1}-z^{k+1}, & a_{4}=v^{k+1}-w^{k+1} \\
a_{5}=v^{k+1}-z^{k+1}, & a_{6}=w^{k+1}-z^{k+1} .
\end{array}
$$

We observe that the first minimization problem in (4) is separable into $n$ subproblems $(j=1, \ldots, n)$ of the form

$$
u_{:, j}^{k+1}=\arg \min _{h \in \mathbb{R}^{m}} \frac{4 \gamma \omega_{c}}{1+6 \mu}\|\nabla h\|_{0}+\left\|h-u_{:, j}^{\prime}\right\|_{2}^{2} .
$$

These are $n$ univariate problems, one for each column. Similarly, the third problem in (4) leads to $m$ univariate problems 
with respect to data $v^{\prime}$, one for each row. The second and fourth minimization problem in (4) lead to univariate problems on the diagonals for data $w^{\prime}$ and $z^{\prime}$, respectively. The crucial point is that all these subproblems are univariate Potts problems which can be solved exactly in quadratic runtime by a method inspired by the dynamic program of [22].

We initialize the ADMM iteration with a small positive coupling parameter $\mu_{0}>0$ and increase it during the iteration by a factor $\tau>1$. Hence, $\mu$ is given by the geometric progression

$$
\mu=\mu_{k}=\tau^{k} \mu_{0}
$$

This strategy assures that, while $u$ and $v$ can evolve quite independently at the beginning of the procedure, they will be close to each other at the end. We stop iterating when the norm of the difference of $u$ and $v$ falls below a certain tolerance.

\section{FEATURE VECTOR OF MONOGENIC CURVELET AMPLITUDES}

In this paper, our feature vectors are defined by monogenic curvelets [16] which are directional wavelets that increase their anisotropy as scale gets finer. They behave like the classical curvelets of [17] at fine scales and like the monogenic wavelets of $[23,24]$ at coarse scales. We give a short description of monogenic curvelets. For details we refer to [16].

The mother curvelet $g_{a}$ at scale $a>0$ is defined via its Fourier transform given by

$$
\widehat{g}_{a}(r, \omega)=W(a r) V\left(\frac{\omega}{\sqrt{a}}\right) .
$$

Here, $(r, \omega)$ denotes polar coordinates, and $W, V$ are radial and angular window functions, respectively; both are compactly supported. The curvelets are such that the finer the scale, the larger the radial bandwidth and the smaller the angular bandwidth. At coarse scales, the angular windowing becomes obsolete and so we set $V \equiv 1$. Let us denote the real part of a curvelet as $\beta_{a 00}=\operatorname{Re} g_{a}$ and let the (real-valued) curvelet family $\beta_{a b \theta}$ be given by

$$
\beta_{a b \theta}(x)=\beta_{a 00}\left(\rho_{\theta}(x-b)\right) .
$$

Here, $\rho_{\theta}$ is a counterclockwise rotation by the angle $\theta$ and $b \in$ $\mathbb{R}^{2}$. The monogenic curvelet coefficient $c_{a b \theta}$ of an image $f$ at scale $a$, at location $b$, and at angle $\theta$ consists of the evaluation of $f$ at $\beta_{a b \theta}$ and their Riesz transforms $\mathcal{R}_{1} \beta_{a b \theta}$ and $\mathcal{R}_{2} \beta_{a b \theta}$ so that

$$
c_{a b \theta}=\left(\left\langle f, \beta_{a b \theta}\right\rangle,\left\langle f, \mathcal{R}_{1} \beta_{a b \theta}\right\rangle,\left\langle f, \mathcal{R}_{2} \beta_{a b \theta}\right\rangle\right) .
$$

The Riesz transform $\mathcal{R}_{\nu}$ is the singular integral operator whose Fourier multiplier is given by $\widehat{\mathcal{R}_{\nu} f}(\xi)=-i \frac{\xi_{\nu}}{|\xi|} \widehat{f}(\xi)$.
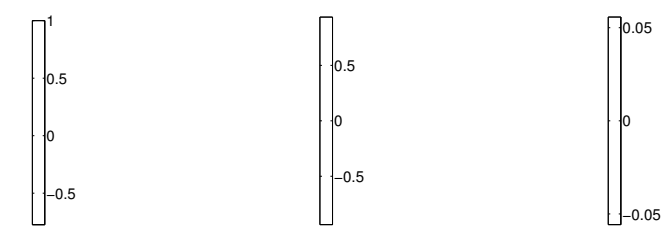

Fig. 2: Monogenic curvelet filters on a fine scale. From left to right: $\beta_{a 00}, \mathcal{R}_{1} \beta_{a 00}$, and $\mathcal{R}_{2} \beta_{a 00}$. (Spatial axes zoomed for reasons of displayability).

Using the monogenic curvelet coefficients $c_{a b \theta}$, we build a feature out of the amplitudes of the coefficients given by

$$
A_{a b \theta}=\sqrt{\left\langle f, \beta_{a b \theta}\right\rangle^{2}+\left\langle f, \mathcal{R}_{1} \beta_{a b \theta}\right\rangle^{2}+\left\langle f, \mathcal{R}_{2} \beta_{a b \theta}\right\rangle^{2}} .
$$

In the discrete setup, we sample the location $b$ at the pixel grid of the image $f$ and the scale at half-octave steps $a_{j}=$ $2^{-j / 2}$. We use 7 octaves, thus, 14 scales in total. At the two finest octaves, we use 8 orientations, at the next two octaves 4 orientations, and so on. Summing all up, the feature vector at each pixel consists of $s=2 \cdot(8+8+4+4+2+2+1)=58$ entries. For color images, we create such a feature vector for all three color channels. We then concatenate the three partial feature vectors which in total gives us feature vectors of length $s=3 \cdot 58=174$.

\section{EXPERIMENTAL RESULTS}

In our experiments, we first create a feature vector of monogenic curvelet amplitudes as described in Section 3. Here we use 58 channels for the gray value images and 174 channels for color images. For the segmentation of the resulting feature vector, we employ the algorithm described in Section 2. In all our experiments, we use $\mu_{k}=0.01 \cdot \gamma \cdot 2^{k}$ for the coupling parameter in (6). The stopping criterion for the algorithm is $\|u-v\|_{2}^{2} \leq \mathrm{TOL} \cdot\|\mathrm{F}\|_{2}^{2}$ with the tolerance $\mathrm{TOL}=10^{-10}$. The model parameter $\gamma$ is chosen empirically.

Note that we do not employ any smoothing steps or nonlinear transform [8] for generating the feature image. In our case, the amplitudes of the monogenic curvelets are already sufficiently smooth and the Potts model provides sufficient regularization so that these steps were not needed.

In Figure 3 we show the application of our method to monochrome and colored texture mosaics ${ }^{1}$. For the two simpler texture patches (first and fourth mosaic), our segmentation almost perfectly gets the boundaries of the groundtruth. The two central images are more challenging but we still obtain satisfactory results.

We present in Figure 1 the segmentation of natural im-

\footnotetext{
${ }^{1}$ The first two texture mosaics are taken from the comparative study [8], the third one from the Prague Texture Segmentation Datagenerator and Benchmark [25], and the fourth one from [26].
} 

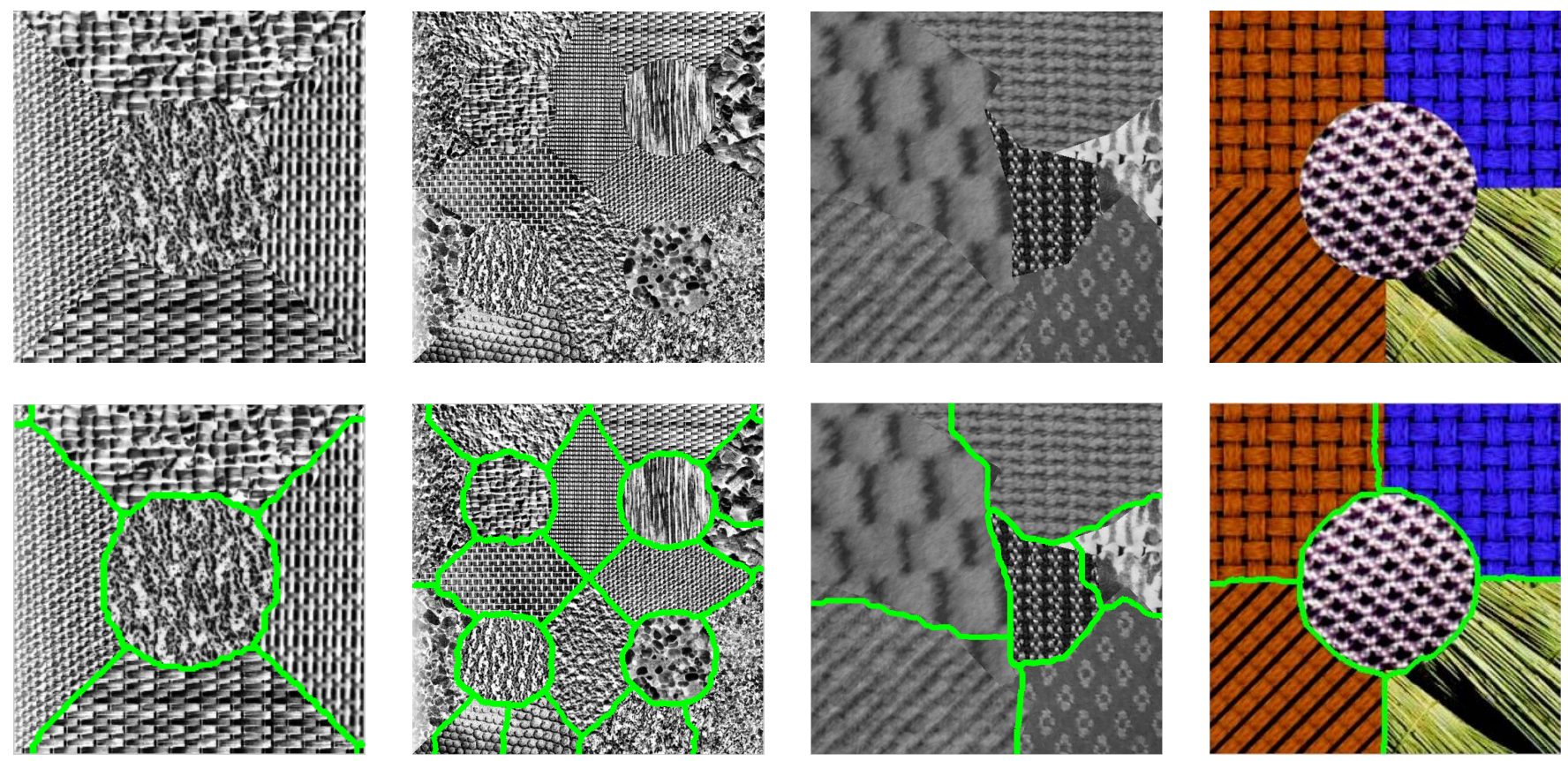

Fig. 3: Top: Texture mosaics. Bottom: Our method using $\gamma=0.14,0.13,0.12$, and 0.8 , respectively. Our method segments the left and the right image almost perfectly. Even on the challenging central images, the major structures are segmented well.

ages $^{2}$. We observe that the differently textured regions are nicely separated.

\section{CONCLUSION}

We have presented a novel approach to the segmentation of textured images. We used feature vectors based on the amplitude of monogenic curvelets. For the segmentation of the high-dimensional feature images, we used a fast computational strategy for the Potts model. Tests carried out on synthetic texture images as well as on real color images show the potential of our approach.

\section{REFERENCES}

[1] M. Yang, W. Moon, Y. Wang, M. Bae, C. Huang, J. Chen, and R. Chang, "Robust texture analysis using multi-resolution gray-scale invariant features for breast sonographic tumor diagnosis," IEEE Transactions on Medical Imaging, vol. 32, no. 12, pp. 2262-2273, 2013.

[2] A. Depeursinge, A. Foncubierta-Rodriguez, D. Van De Ville, and H. Müller, "Three-dimensional solid texture analysis in biomedical imaging: Review and opportunities," Medical Image Analysis, vol. 18, no. 1, pp. 176 - 196, 2014.

[3] J. Shotton, J. Winn, C. Rother, and A. Criminisi, “Textonboost for image understanding: Multi-class object recognition and segmentation by jointly modeling texture, layout, and context,"

${ }^{2}$ The images were taken from titanic-magazin.de, zastavki.com, 123rf.com.
International Journal of Computer Vision, vol. 81, no. 1, pp. 223, 2009.

[4] M. Pietikäinen, Computer vision using local binary patterns, Springer London, 2011.

[5] R. Haralick, "Statistical and structural approaches to texture," Proceedings of the IEEE, vol. 67, no. 5, pp. 786-804, 1979.

[6] A. Jain and F. Farrokhnia, "Unsupervised texture segmentation using Gabor filters," Pattern Recognition, vol. 24, no. 12, pp. 1167-1186, 1991.

[7] M. Unser, "Texture classification and segmentation using wavelet frames," IEEE Transactions on Image Processing, vol. 4, no. 11, pp. 1549-1560, 1995.

[8] T. Randen and J. Husoy, "Filtering for texture classification: A comparative study," IEEE Transactions on Pattern Analysis and Machine Intelligence, vol. 21, no. 4, pp. 291-310, 1999.

[9] M. Rousson, T. Brox, and R. Deriche, "Active unsupervised texture segmentation on a diffusion based feature space," in Proceedings of the IEEE Conference on Computer Vision and Pattern Recognition, Madison, 2003, vol. 2, pp. II-699.

[10] M. Ozden and E. Polat, "Image segmentation using color and texture features," in Proceedings of the 13th European Signal Processing Conference, Antalya, 2005, pp. 2226-2229.

[11] J. Santner, M. Unger, T. Pock, C. Leistner, A. Saffari, and H. Bischof, "Interactive texture segmentation using random forests and total variation," in British Machine Vision Conference, 2009, pp. 1-12.

[12] S. Geman and D. Geman, "Stochastic relaxation, Gibbs distributions, and the Bayesian restoration of images," IEEE Transactions on Pattern Analysis and Machine Intelligence, vol. 6, no. 6, pp. 721-741, 1984. 
[13] D. Mumford and J. Shah, "Optimal approximations by piecewise smooth functions and associated variational problems," Communications on Pure and Applied Mathematics, vol. 42, no. 5, pp. 577-685, 1989.

[14] Y. Boykov, O. Veksler, and R. Zabih, "Fast approximate energy minimization via graph cuts," IEEE Transactions on Pattern Analysis and Machine Intelligence, vol. 23, no. 11, pp. 12221239, 2001.

[15] Z. Kato and T.-C. Pong, "A Markov random field image segmentation model for color textured images," Image and Vision Computing, vol. 24, no. 10, pp. 1103-1114, 2006.

[16] M. Storath, "Directional multiscale amplitude and phase decomposition by the monogenic curvelet transform," SIAM Journal on Imaging Sciences, vol. 4, no. 1, pp. 57-78, 2011.

[17] E. Candès and D. Donoho, "Continuous curvelet transform: I. Resolution of the wavefront set," Applied and Computational Harmonic Analysis, vol. 19, no. 2, pp. 162-197, 2005.

[18] L. Semler and L. Dettori, "Curvelet-based texture classification of tissues in computed tomography," in IEEE International Conference on Image Processing, 2006, pp. 2165-2168.

[19] S. Arivazhagan, L. Ganesan, and T. Kumar, "Texture classification using curvelet statistical and co-occurrence features," in 18th International Conference on Pattern Recognition, 2006, vol. 2, pp. 938-941.

[20] M. Felsberg and G. Sommer, "The monogenic signal," IEEE Transactions on Signal Processing, vol. 49, no. 12, pp. 31363144, 2001.

[21] S. Boyd, N. Parikh, E. Chu, B. Peleato, and J. Eckstein, "Distributed optimization and statistical learning via the alternating direction method of multipliers," Foundations and Trends in Machine Learning, vol. 3, no. 1, pp. 1-122, 2011.

[22] F. Friedrich, A. Kempe, V. Liebscher, and G. Winkler, "Complexity penalized M-estimation," Journal of Computational and Graphical Statistics, vol. 17, no. 1, pp. 201-224, 2008.

[23] M. Unser, D. Sage, and D. Van De Ville, "Multiresolution monogenic signal analysis using the Riesz-Laplace wavelet transform," IEEE Transactions on Image Processing, vol. 18, no. 11, pp. 2402-2418, 2009.

[24] S. Held, M. Storath, P. Massopust, and B. Forster, "Steerable wavelet frames based on the Riesz transform," IEEE Transactions on Image Processing, vol. 19, no. 3, pp. 653-667, 2010.

[25] M. Haindl and S. Mikes, "Texture segmentation benchmark," in Proceedings of the IEEE International Conference on Pattern Recognition, Los Alamitos, 2008, pp. 1-4.

[26] M. Hoang, J.-M. Geusebroek, and A. Smeulders, "Color texture measurement and segmentation," Signal Processing, vol. 85, no. 2, pp. 265-275, 2005. 